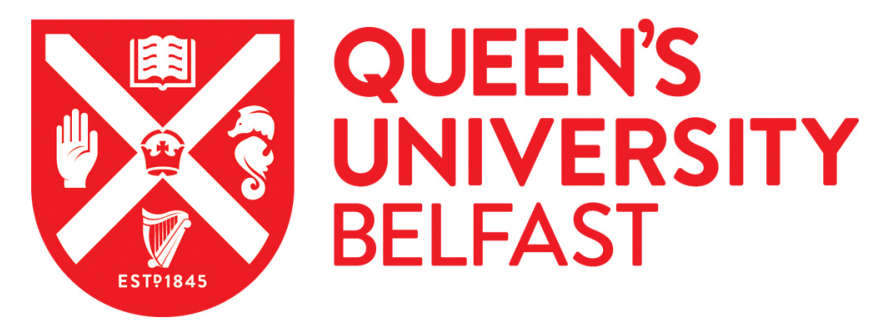

\title{
Infrared emission from tunneling electrons: the end of the rainbow in scanning tunneling microscopy
}

Boyle, M. G., Mitra, J., \& Dawson, P. (2009). Infrared emission from tunneling electrons: the end of the rainbow in scanning tunneling microscopy. Applied Physics Letters, 94(23), [233118]. https://doi.org/10.1063/1.3154563

Published in:

Applied Physics Letters

Document Version:

Publisher's PDF, also known as Version of record

Queen's University Belfast - Research Portal:

Link to publication record in Queen's University Belfast Research Portal

Publisher rights

Copyright 2009 American Institute of Physics.

This work is made available online in accordance with the publisher's policies. Please refer to any applicable terms of use of the publisher.

\section{General rights}

Copyright for the publications made accessible via the Queen's University Belfast Research Portal is retained by the author(s) and / or other copyright owners and it is a condition of accessing these publications that users recognise and abide by the legal requirements associated with these rights.

Take down policy

The Research Portal is Queen's institutional repository that provides access to Queen's research output. Every effort has been made to ensure that content in the Research Portal does not infringe any person's rights, or applicable UK laws. If you discover content in the Research Portal that you believe breaches copyright or violates any law, please contact openaccess@qub.ac.uk. 


\title{
Infrared emission from tunneling electrons: The end of the rainbow in scanning tunneling microscopy
}

\author{
Michael G. Boyle, J. Mitra, and P. Dawson ${ }^{\text {a) }}$ \\ Centre for Nanostructured Media, School of Mathematics and Physics, Queen's University of Belfast, \\ Belfast BT7 1NN, United Kingdom
}

(Received 13 February 2009; accepted 26 May 2009; published online 12 June 2009)

\begin{abstract}
Electromagnetic radiation originating with localized surface plasmons in the metal-tip/metal-sample nanocavity of a scanning tunneling microscope is demonstrated to extend to a wavelength $\lambda$ of at least $1.7 \mu \mathrm{m}$. Progressive spectral extension beyond $\lambda \sim 1.0 \mu \mathrm{m}$ occurs for increasing tip radius above $\sim 15 \mathrm{~nm}$, reaching $\lambda \sim 1.7 \mu \mathrm{m}$ for tip radius $\sim 100 \mathrm{~nm}$; these observations are corroborated by use of a simple physical model that relates the discrete plasmon mode frequencies to the tip radius. This spectral extension opens up a new regime for scanning tunneling microscope-based optical spectroscopy. (C) 2009 American Institute of Physics. [DOI: 10.1063/1.3154563]
\end{abstract}

Light emission from the electron scanning tunneling microscope (LESTM) was first reported over $20 \mathrm{yr}$ ago from both metallic and semiconductor samples. ${ }^{1}$ The attraction of LESTM is that the spatial resolution of STM is complemented with the analytical capability of optical spectroscopy. Focusing on the metal tip/metal sample configuration addressed here, there have followed spectroscopic studies of the light emitted from planar samples ${ }^{2-5}$ and a variety of nanoscale entities, ${ }^{6-9}$ extending to fluorescent molecules in the tunnel gap. ${ }^{10}$ However, in the cited work spectra extend only to the long-wavelength detection limit of Si-based detectors, $\lambda \sim 1.0 \mu \mathrm{m}$. Here, emission in the short-wave infrared (SWIR) is explored where we define this regime as 1.0 $<\lambda<1.7 \mu \mathrm{m}$, the upper limit being set by the InGaAs detector used. This offers significant extension to the spectral range for LESTM, including the optical communications wavelengths, and facilitates more comprehensive characterization of a broad range of metallic and other nanoentities. In addition, it is highly pertinent to the converse configuration of tip-enhanced Raman scattering, ${ }^{11}$ while extension further into the IR could open up the possibility of direct STMbased molecular spectroscopy.

Early experimental work $^{1,2}$ and much theoretical analysis $^{3,12-14}$ on the metal tip/metal sample system established that the light emission is due to the excitation and subsequent radiative decay of localized surface plasmon (LSP) modes of the tip-sample nanocavity. These comprise a set of optical frequency, coupled charge oscillations between tip and sample and occur at discrete energies determined by the cavity geometry, notably the tip profile, and by the optical properties of tip and sample. Intrinsic damping leads to spectral broadening, resulting in a continuous broadband output with peaks that correspond to the LSP modal energies. The optical frequency fluctuations in the tunnel current that drive the LSP modes have a power spectral density function $C(\omega) \propto\left(1-\hbar \omega / e V_{b}\right)$, where $\omega$ is the angular frequency and $V_{b}$ is the applied bias; ${ }^{14,15}$ the function applies in the regime $\hbar \omega \leq e V_{b}$, the equality denoting a quantum cutoff condition that determines the short-wavelength spectral limit. The question this Letter addresses is what is the long wavelength limit of the emission (where is the other end of the STM

${ }^{a)}$ Electronic mail: p.dawson@qub.ac.uk. rainbow?) and what are the factors that determine it.

At the outset we also emphasize that the key LESTM results reported here are combined with explicit, independent characterization of the tip profiles from scanning electron microscopy (SEM); this information is critical to the interpretation of the spectra. It is surprising that only one previous investigation ${ }^{4}$ has offered such information systematically. For this study, both tip and sample were made of $\mathrm{Au}$, the only metal of the requisite optical properties for good emission that may be used in ambient without rapid contamination. Both mechanically cut and electrochemically etched Au tips ${ }^{16}$ were formed in order to offer a range of tip size; thin films of polycrystalline $\mathrm{Au}$ (rms roughness $<1.5 \mathrm{~nm}$ ) or flame annealed $\mathrm{Au}(111)$ were used as samples. A Digital Instruments Nanoscope E (configured for STM operation) was used, with each experimental run lasting typically $300 \mathrm{~s}$. Light was collected by two low-OH content $800 \mu \mathrm{m}$ core optical fibers, placed $\sim 1 \mathrm{~mm}$ from the tip-sample junction, and analyzed by spectrographs equipped with an InGaAs photodiode array (range $0.80<\lambda<1.70 \mu \mathrm{m}$ ) and a $\mathrm{Si}$ charge coupled device (CCD) camera $(0.40<\lambda<1.05 \mu \mathrm{m})$. SEM images of the tips were taken immediately before and after the acquisition of several consistent, stable spectra (in-

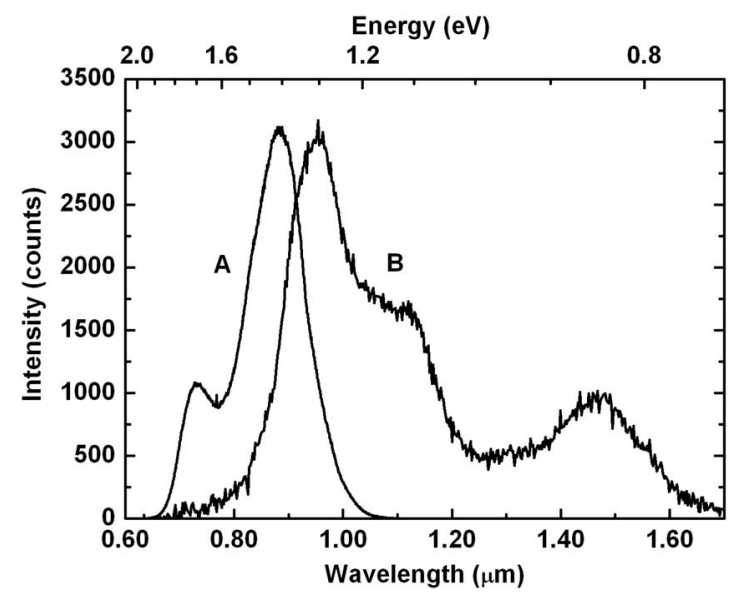

FIG. 1. Emission spectra recorded from STM with blunt Au tip and Au(111) sample using A Si CCD array and B InGaAs photodiode array. The main peak in $A$ is scaled to a count of $\sim 3000$ for direct comparison with the same peak in B. $V_{b}=1.85 \mathrm{~V} ; i_{t}=20 \mathrm{nA}$, scan range is $50 \times 50 \mathrm{~nm}^{2}$. 


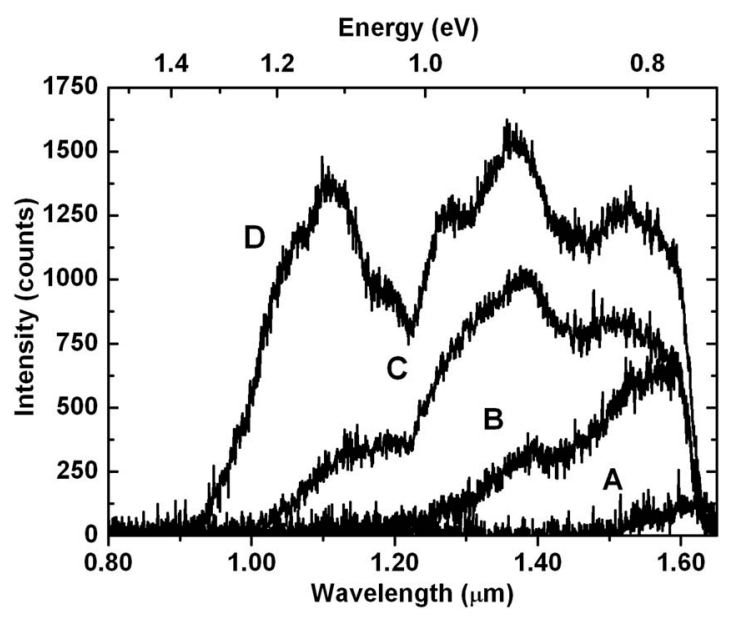

FIG. 2. IR emission spectra in wavelength range of $0.80-1.65 \mu \mathrm{m}$ (energy range of $1.55-0.75 \mathrm{eV}$ ) with bias, $V_{b}$, A $0.8 \mathrm{~V}, \mathrm{~B} 1.0 \mathrm{~V}, \mathrm{C} 1.2 \mathrm{~V}$, and D 1.4 $\mathrm{V}$ and constant tunnel current $i_{t}=40 \mathrm{nA}$. Scan range is $100 \times 100 \mathrm{~nm}^{2}$.

dicating stable tip conditions). In Fig. 1 spectra A and B were acquired simultaneously with the $\mathrm{Si}$ and InGaAs devices, respectively, there is clearly significant SWIR emission. The tip was formed using a rapid-etch technique to deliberately yield a blunt end-profile in order to enhance the SWIR component, as explained below. The spectra are uncorrected for system spectral response which shifts the peaks by $<10 \mathrm{~nm}$ across most of the spectral range $(1.0<\lambda<1.60 \mu \mathrm{m})$.

Figure 2 shows emission spectra from a mechanically cut $\mathrm{Au}$ tip/polycrystalline $\mathrm{Au}$ sample for various values of bias $V_{b}$ at constant tunnel current $i_{t}$. There are three features of note. First, there is good conformity with the quantum cutoff condition. Second, the principal spectral features remain essentially constant in wavelength with increasing bias, indicating a consistent and stable STM setup over the bias range. Third, the abrupt nature of the long-wavelength decay is probably due to detector cutoff, suggesting extension of the emission even further into the IR.

We next outline an understanding of the spectral output in terms of the Rendell and Scalapino (RS) model, ${ }^{17}$ with salient results, summarized in Fig. 3, used to interpret the critical experimental data of Fig. 4. The RS model gives an analytic expression for the LSP modal frequencies for a metal sphere of radius $R$, separated from a semi-infinite, planar metal electrode by a distance $d$ in a medium of dielectric constant $\varepsilon_{a}$

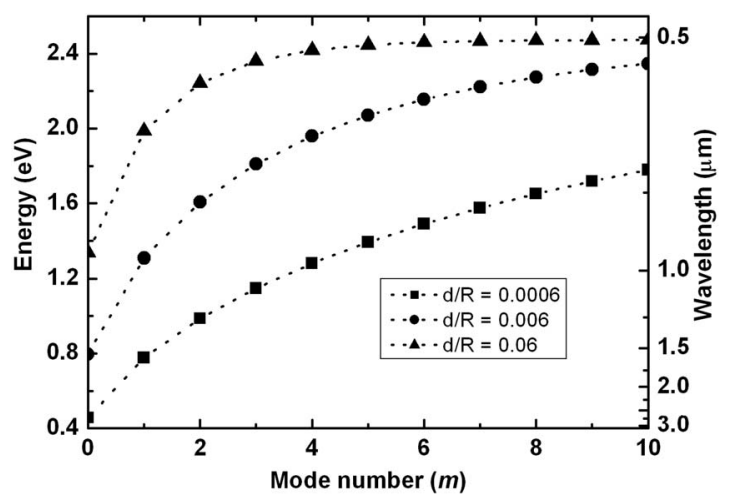

FIG. 3. Energy (wavelength) versus mode number for LSP modes evaluated from Eq. (1) with $\omega_{p} \sim 5.32 \times 10^{15} \mathrm{rad} \mathrm{s}^{-1}$ and various ratios of tunnel gap to tip radius $d / R$. Dashed lines are a guide to the eye.
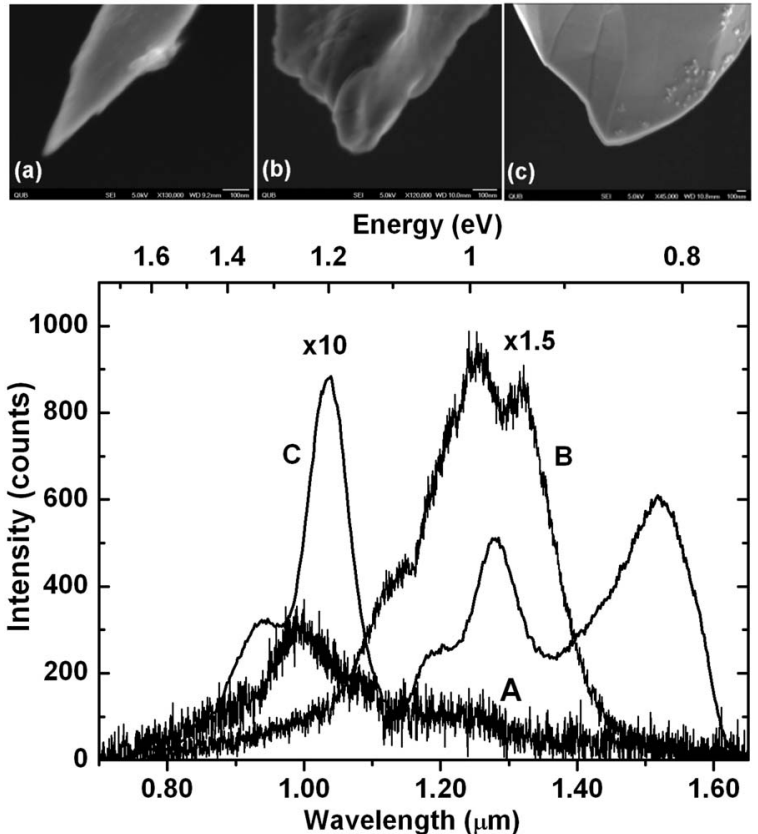

FIG. 4. SEM images $[(a)-(c)]$ of three Au tips of contrasting radii, taken after acquisition of spectra and (d), corresponding (A-C) IR emission spectra from $\mathrm{Au}(111)$ surface. For $\mathrm{A}$ and $\mathrm{B}, V_{b}=1.6 \mathrm{~V}, i_{t}=40 \mathrm{nA} ; \mathrm{C}$ $V_{b}=1.8 \mathrm{~V}, i_{t}=20 \mathrm{nA}$; scan range is $20 \times 20 \mathrm{~nm}^{2}$.

$$
\omega_{m}=\omega_{p}\left[\frac{\tanh \left(m+\frac{1}{2}\right) \beta_{0}}{\varepsilon_{a}+\tanh \left(m+\frac{1}{2}\right) \beta_{0}}\right]^{1 / 2} \quad m=0,1,2, \ldots,
$$

where $\omega_{p}$ is the bulk plasmon frequency of the substrate metal and $\beta_{o}=\operatorname{arccosh}(1+d / R)$. Essentially, it identifies resonant frequencies for which a half-wavelength of the particle-surface interface modes fit into the cavity between the two. Assumptions underpinning Eq. (1) should be noted in relation its application to a STM tip-sample junction. First, mode confinement is largely to the underside of the sphere, within a region defined by a tip-sample separation that is approximately twice the smallest tip-sample distance $d$ leading to a characteristic localization length $L \approx \sqrt{2 R d} ; ;^{14,15}$ this assumes $R \gg d$ which is indeed the case in STM. Second, the tip metal is assumed to be ideal $\left(\varepsilon_{t}=-\infty\right)$ and the substrate to be a free electron metal, i.e., $\varepsilon_{s}=\varepsilon_{s-r}+i \varepsilon_{s-i}=\left(1-\omega_{p}^{2} / \omega^{2}\right)+i 0$; the issue of appropriate values for the optical data for Au will be duly discussed.

In applying the RS model to the data, we build on our detailed study of emission in the visible-near-IR regime ${ }^{17}$ in which the spectral peak positions are very successfully described by Eq. (1) using $\omega_{p}=5.4 \pm 0.1 \times 10^{15} \mathrm{rad} \mathrm{s}^{-1}$ and $d$ $=0.55 \pm 0.05 \mathrm{~nm}$, with the constraint that $R$ must be closely similar to the experimentally determined tip radius $R_{\mathrm{SEM}}$. Our motivation to use the RS model stems from the fact that correlation between tip radius and spectral output is actually problematic in the current line of detailed modeling of the emission process. ${ }^{3,12-14}$ Specifically, as Meguro et al. ${ }^{4}$ point out, unrealistically sharp tips are required to obtain LSP modes that (a) are sufficiently closely spaced in energy to coincide with the observed spectral peak spacing and (b) extend even into the near-IR; no SWIR emission is predicted for physically feasible tip structures. We note also that the tip dimension is the issue of primary importance in determining the spectral output, with the detail of the shape being secondary. 
It is now instructive to consider the implications of Fig. 3 which shows the energies/wavelengths of the discrete LSP modes as a function of mode number, evaluated from Eq. (1), using $\omega_{p} \sim 5.32 \times 10^{15} \mathrm{rad} \mathrm{s}^{-1}$ and various ratios of $d / R$, $0.06,0.006$, and 0.0006 , corresponding to $R=10,100$, and $1000 \mathrm{~nm}$ for $d=0.6 \mathrm{~nm}$. Note, in particular, that for the two larger radii, the lowest order modes extend significantly into the IR. The corollary is that for very sharp tips $(R=10 \mathrm{~nm})$ the LSP modes are confined to shorter wavelengths and SWIR emission should be extinguished.

Figure 4, which presents three contrasting tip profiles and their corresponding LESTM spectra, forms a critical test of the understanding outlined above. For the sharpest tip [Fig. 4(a), $R_{\mathrm{SEM}} \sim 15 \mathrm{~nm}$ ] spectrum A shows a single dominant peak at $\sim 0.995 \mu \mathrm{m}$ with residual emission extending to $1.3 \mu \mathrm{m}$, but no output beyond that to the detector cutoff. Using $\omega_{p} \sim 5.32 \times 10^{15} \mathrm{rad} \mathrm{s}^{-1}$ (this value is used throughout) $d=0.55 \mathrm{~nm}$ and $R=14 \mathrm{~nm}$, Eq. (1) yields $\lambda_{0}$ $=0.989 \mu \mathrm{m}$ (i.e., $m=0$ mode), with $\lambda_{1}=0.648 \mu \mathrm{m}$ which lies outside the InGaAs detection window. Tip 4(b) with $R_{\mathrm{SEM}} \sim 48 \mathrm{~nm}$ yields spectrum $\mathrm{B}$, which displays a double peak structure at 1.253 and $1.315 \mu \mathrm{m}$ and effectively drops to zero for $\lambda>1.50 \mu \mathrm{m}$. For simplicity, treating the spectrum as having a single-peak associated with a uniquely defined tip radius, Eq. (1) yields $\lambda_{0}=1.284 \mu \mathrm{m}$ (an average of the two peak wavelengths) for input values $d=0.55 \mathrm{~nm}$ and $R=45 \mathrm{~nm}$, while $\lambda_{1}=0.798 \mu \mathrm{m}$. Spectrum $\mathrm{C}$, acquired with a blunt, mechanically cut tip $\left[R_{\mathrm{SEM}} \sim 100 \mathrm{~nm}\right.$, Fig. $\left.4(\mathrm{c})\right]$, is multifeatured (peaks at 1.040, 1.285, and $1.520 \mu \mathrm{m}$ and shoulders at 1.200 and $0.920 \mu \mathrm{m}$ ) and crucially extends right across the spectral detection window to the longwavelength detector cutoff. The modal designation of the spectral features is less straightforward in this case; using $R=94 \mathrm{~nm}$ and $d=0.55 \mathrm{~nm}$, Eq. (1) yields $\lambda_{0}=1.525 \mu \mathrm{m}$ and $\lambda_{1}=0.928 \mu \mathrm{m}$ (and $\lambda_{2}=0.775 \mu \mathrm{m}$ ), corresponding closely with two of the spectral features. In order to generate LSP modes mapping to the remaining spectral structure we suggest a contribution from a second dominant component of the tip profile with $R=400 \mathrm{~nm}$, yielding LSP modes at $\lambda_{1}$ $=1.294 \mu \mathrm{m}, \lambda_{2}=1.027 \mu \mathrm{m}$, and $\lambda_{3}=0.890 \mu \mathrm{m}$. This is suggested by the ridge feature seen in the SEM tip image running approximately orthogonal to the in-plane profile with a protrusion back from the focal plane of the image, indicating a flattened tip structure in this direction. Finally, it is also notable that the emission intensity increases with $R$ due to the greater coupling area available on the tip which scales according to $\sqrt{2 R d}$; detailed calculation of the emission predicts a significant increase of intensity with tip radius. ${ }^{14}$

Lastly, some comment on the properties of $\mathrm{Au}$ is warranted. Although the value of $\hbar \omega_{p}=3.5 \mathrm{eV} \quad\left(\omega_{p}=5.32\right.$ $\times 10^{15} \mathrm{rad} \mathrm{s}^{-1}$ ) used here is significantly lower than the generally accepted value of $\sim 9.0 \mathrm{eV}$ for bulk gold, we regard it as physically realistic for several reasons. First, reduction in the mean free path of conduction electrons due to grain boundary and surface scattering in thin films leads to a lower effective value for $\hbar \omega_{p}$, as has been clearly demonstrated in a recent study of thin Au films. ${ }^{18}$ Clearly, this mean-free-path effect will be more significant in the confined geometry of an STM tip. Second, screening effects come significantly into play with two metallic bodies in close proximity which may be treated equivalently as an effective reduction in the density of free electrons available to participate in plasmons oscillations ${ }^{19}$ or, in other words, a reduction in $\hbar \omega_{p}$; consideration of this effect has recently been extended to the tunneling regime. ${ }^{20}$ Finally and importantly are the consequences of the extreme conditions of the tunneling environment (electric field of $\sim 4 \times 10^{9} \mathrm{~V} \mathrm{~m}^{-1}$ and current density of $\sim 10 \mathrm{GA} / \mathrm{m}^{2}$ ), which tend toward those of field ionization that lead to material transfer between tip and sample. $^{21,22}$ There is significant local distortion of the background lattice and lowering of the interatomic potentials at the tip end and immediately below, on the sample surface. ${ }^{22}$ The corollary of lower electron density, reduced electronic restoring forces and increased electron scattering (a significant increase of $\varepsilon_{\mathrm{Au}-i}$ reduces $\left|\varepsilon_{\mathrm{Au}-r}\right|$ in modified Drude models) all combine to yield an effective local lowering of $\hbar \omega_{p}$.

In conclusion, the emission of electromagnetic radiation from STM up to $\lambda \sim 1.70 \mu \mathrm{m}$ has been demonstrated for the case of tunneling between a relatively blunt $(R \sim 100 \mathrm{~nm})$ $\mathrm{Au}$ tip and Au sample, with a progressive confinement of the emission to shorter wavelengths as the tip is made sharper. This is explained in terms of the identification of spectral peaks with LSP modal energies calculated from the RS model, ${ }^{15}$ with good agreement between the measured and model values of tip radii, but with a lowered value of $\hbar \omega_{p}$. The observation of SWIR emission should open up a new vista for optical STM studies of nanoentities and molecules.

The authors are grateful to the Engineering and Physical Sciences Research Council (U.K.) for supporting this research through Grant No. EP/D048850/1.

${ }^{1}$ J. H. Coombs, J. K. Gimzewski, B. Reihl, J. K. Sass, and R. R. Schlittler, J. Microsc. 152, 325 (1988).

${ }^{2}$ R Berndt, J. K. Gimzewski, and P. Johansson, Phys. Rev. Lett. 67, 3796 (1991); 71, 3493 (1993).

${ }^{3}$ J. Aizpurua, S. P. Apell, and R. Berndt, Phys. Rev. B 62, 2065 (2000).

${ }^{4}$ K. Meguro, K. Sakamoto, R. Arafune, M. Satoh, and S. Ushioda, Phys. Rev. B 65, 165405 (2002).

${ }^{5}$ P. Dawson and M. G. Boyle, J. Opt. A, Pure Appl. Opt. 8, S219 (2006).

${ }^{6}$ A. Downes and P. Dumas, Appl. Surf. Sci. 212, 770 (2003).

${ }^{7}$ M. G. Boyle, J. Mitra, and P. Dawson, Jpn. J. Appl. Phys., Part 1 45, 2119 (2006)

${ }^{8}$ G. V. Nazin, X. H. Qiu, and W. Ho, Phys. Rev. Lett. 90, 216110 (2003).

${ }^{9}$ G. Hoffmann, T. Maroutian, and R. Berndt, Phys. Rev. Lett. 93, 076102 (2004).

${ }^{10}$ X.-L. Guo, Z.-C. Dong, and A. S. Trifinov, Appl. Phys. Lett. 84, 969 (2004).

${ }^{11}$ J. Steidtner and B. Pettinger, Phys. Rev. Lett. 100, 236101 (2008).

${ }^{12}$ P. Johansson, R. Monreal, and P. Apell, Phys. Rev. B 42, 9210 (1990).

${ }^{13}$ Y. Uehara, Y. Kimura, S. Ushioda, and K. Takeuchi, Jpn. J. Appl. Phys., Part 1 31, 2465 (1992).

${ }^{14}$ P. Johansson, Phys. Rev. B 58, 10823 (1998).

${ }^{15}$ R. W. Rendell and D. J. Scalapino, Phys. Rev. B 24, 3276 (1981).

${ }^{16}$ M. G. Boyle, L. Feng, and P. Dawson, Ultramicroscopy 108, 558 (2008).

${ }^{17}$ M. G. Boyle, Ph.D. thesis, Queen's University of Belfast, 2005.

${ }^{18}$ V. B. Svetovoy, P. J. van Zwol, G. Palasantzas, and J. Th. M. Hosson, Phys. Rev. B 77, 035439 (2008).

${ }^{19}$ F. Le, N. Z. Lwin, J. M. Steele, M. Kall, N. J. Halas, and P. Nordlander, Nano Lett. 5, 2009 (2005).

${ }^{20}$ J. Zuloaga, E. Prodan, and P. Nordlander, Nano Lett. 9, 887 (2009).

${ }^{21}$ H. J. Mamin, P. H. Guethner, and D. Rugar, Phys. Rev. Lett. 65, 2418 (1990).

${ }^{22}$ T. T. Tsong, Phys. Rev. B 44, 13703 (1991). 